\title{
Super persistent transient in a master-slave configuration with Colpitts oscillators
}

\author{
R. C. Bonetti ${ }^{1}$, S. L. T. de Souza ${ }^{2}$, A. M. Batista ${ }^{3}$, J. D. \\ Szezech Jr. ${ }^{3}$, I. L. Caldas ${ }^{4}$, R. L. Viana ${ }^{5}$, S. R. Lopes $^{5}$, M. S. \\ Baptista $^{6}$ \\ 1 Pós-Graduação em Ciências, Universidade Estadual de Ponta Grossa, 84030-900, \\ Ponta Grossa, Paraná, Brazil \\ ${ }^{2}$ Departamento de Física e Matemática, Universidade Federal de São João del Rei, \\ 36420-000, Ouro Branco, Minas Gerais, Brazil \\ ${ }^{3}$ Departamento de Matemática e Estatística, Universidade Estadual de Ponta Grossa, \\ 84030-900, Ponta Grossa, Paraná, Brazil \\ ${ }^{4}$ Instituto de Física, Universidade de São Paulo, Caixa Postal 66316, 05315-970, São \\ Paulo, SP, Brazil \\ ${ }^{5}$ Departamento de Física, Universidade Federal do Paraná, 81531-990, Curitiba, \\ Paraná, Brazil \\ ${ }^{6}$ Institute for Complex Systems and Mathematical Biology, SUPA, University of \\ Aberdeen, AB24 3UE Aberdeen, United Kingdom \\ E-mail: ${ }^{3}$ antoniomarcosbatista@gmail.com
}

\begin{abstract}
Master-slave systems have been intensively investigated to model the application of chaos to communications. We considered Colpitts oscillators coupled according to a master-slave configuration to study chaos synchronisation. We revealed the existence of super persistent transients in this coupled system. Moreover, we showed that an additive noise added to the slave system may suppress chaos synchronisation. When synchronisation is not suppressed, the noise induces longer transients.
\end{abstract}

Keywords: Colpitts, synchronisation, master-slave

PACS numbers: 05.45.-a,05.45.Xt 


\section{Introduction}

Coupled chaotic systems can synchronise their trajectories [1]. The synchronisation of coupled chaotic systems has important applications in many fields, such as: biological systems [2], secure communications [3], chemical reactions [4], etc.

Recent works have shown that chaos synchronisation of a master-slave configuration is relevant to communication systems. The slave system is driven by a signal derived from the master [5]. Here we focus the study in two chaotic coupled Colpitts oscillators [6]. We choose this circuit due to the fact that it can be useful in applications to communication systems, as well as, it exhibits a rich dynamical behaviour for certain parameter values [7]. In the Colpitts oscillator the operation frequency can vary from few Hertz up to the microwave frequency range, a characteristic that enables the use of this circuit to transmit information in channels with difference frequency bandwidths.

There has been a great interest in the study of synchronisation and control of Colpitts oscillators. Control schemes were used to suppress chaos. Li and collaborators used a controller to drive chaotic Colpitts to a desired state [8], that is, the stabilisation of the chaotic motion to a steady state. In another work, the circuit was controlled by using a non linear feedback [9]. It has been found synchronisation between chaotic Colpitts in identical and mismatched cases [10, 11]. Furthermore, there are works about synchronisation of Colpitts oscillators that operate in ultra high frequency range [12].

In this article, we study two coupled Colpitts oscillators in a master-slave configuration and focus our attention to chaos synchronisation. Our main objective is to verify the existence of super persistent chaotic transients [13, 14], and the effect of noise on the synchronous behaviour [15, 16].

This article is organized as follows: in section 2 we present the coupled Colpitts oscillators. In section 3 we study the onset of synchronisation and show the existence of super persistent transients. In section 4 we describe the effect of noise on the system. The last section contains our conclusions.

\section{Colpitts oscillator}

The Colpitts oscillator is a type of resonant circuit with a transistor for feedback. This oscillator has been used in electronic devices and communication systems, due to the fact that it can exhibit chaos [17]. Fig. 11(a) exhibits the circuit configuration containing a bipolar junction transistor (BJT) according to Fig. 1(b). The state equations are given by

$$
\begin{aligned}
& C_{1} \frac{d V_{C_{1}}}{d t^{\prime}}=-\alpha_{F} f\left(-V_{C_{2}}\right)+I_{L}, \\
& C_{2} \frac{d V_{C_{2}}}{d t^{\prime}}=\left(1-\alpha_{F}\right) f\left(-V_{C_{2}}\right)-G_{0} V_{C_{2}}+I_{L}-I_{0}, \\
& L \frac{d I_{L}}{d t^{\prime}}=-V_{C_{1}}-V_{C_{2}}-R I_{L}+V_{C C},
\end{aligned}
$$




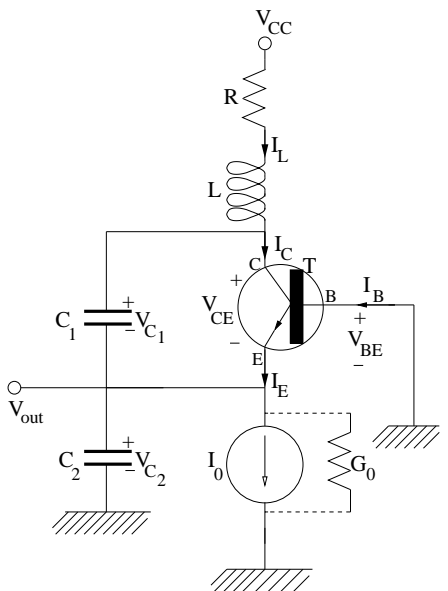

(a)

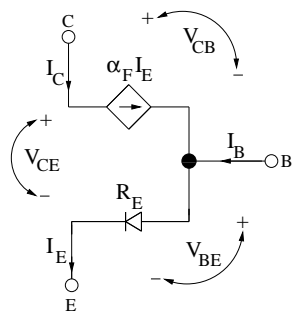

(b)

Figure 1. The circuit diagram of a Colpitts oscillator. (a) Circuit configuration and (b) the bipolar junction transistor (BJT).

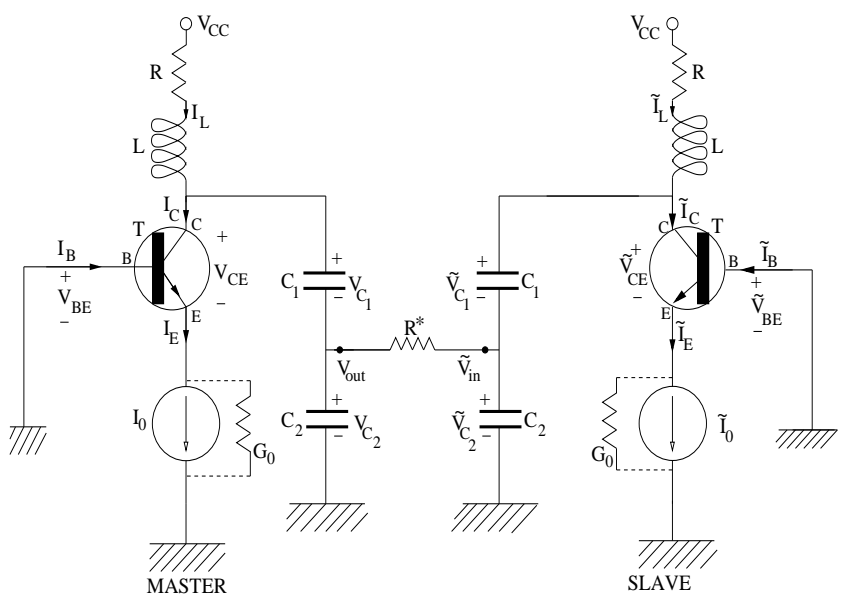

Figure 2. The circuit diagram of coupled Colpitts with a master-slave configuration.

where the voltages $V_{C_{1}}$ and $V_{C_{2}}$ are associated with the capacitors $C_{1}$ and $C_{2}$, respectively, $V_{c c}$ is the voltage supply, $I_{L}$ is the current through the inductor $L$ and $t$ is the time. There is a current generator $I_{0}$ to maintain constant biasing emitter current. The function $f()$ is the driving-point characteristic of the non linear resistor $R_{E}$ and it can be expressed as $I_{E}=f\left(V_{C_{2}}\right)=f\left(-V_{B E}\right)$, where $\alpha_{F}$ is the common-base forward short-circuit gain.

We consider two Colpitts oscillators in a master-slave configuration, in accordance with the scheme showed by Fig. 2. If this coupled system would be used for communication purposes, the transmitter would be the master and the receiver the slave. We also consider noise in the channel.

Introducing the following dimensionless variables

$$
x_{1}=\frac{V_{C_{1}}-\bar{V}_{C_{1}}}{V_{T}}
$$




$$
\begin{aligned}
& x_{2}=\frac{V_{C_{2}}-\bar{V}_{C_{2}}}{V_{T}}, \\
& x_{3}=\frac{I_{L}-\bar{I}_{L}}{I_{0}},
\end{aligned}
$$

where

$$
\begin{aligned}
& \bar{V}_{C_{1}}=V_{C C}-\alpha_{F} R f\left(-\bar{V}_{C_{2}}\right)-\bar{V}_{C_{2}}, \\
& \bar{V}_{C_{2}}=\frac{1}{G_{0}}\left[n\left(-\bar{V}_{C_{2}}\right)-I_{0}\right] \\
& \bar{I}_{L}=-\alpha_{F} f\left(-\bar{V}_{C_{2}}\right),
\end{aligned}
$$

we obtain the equations of an unidirectional master-slave configuration

$$
\begin{aligned}
\frac{d x_{1}}{d t} & =\frac{g}{Q(1-k)}\left[-\alpha_{F} n\left(x_{2}\right)+x_{3}\right], \\
\frac{d x_{2}}{d t} & =\frac{g}{Q k}\left[\left(1-\alpha_{F}\right) n\left(x_{2}\right)+x_{3}\right], \\
\frac{d x_{3}}{d t} & =-\frac{Q k(1-k)}{g}\left[x_{1}+x_{2}\right]-\frac{1}{Q} x_{3}, \\
\frac{d y_{1}}{d t} & =\frac{g}{Q(1-k)}\left[-\alpha_{F} n\left(y_{2}\right)+y_{3}\right], \\
\frac{d y_{2}}{d t} & =\frac{g}{Q k}\left[\left(1-\alpha_{F}\right) n\left(y_{2}\right)+y_{3}\right]+\varepsilon\left[x_{2}-y_{2}\right], \\
\frac{d y_{3}}{d t} & =-\frac{Q k(1-k)}{g}\left[y_{1}+y_{2}\right]-\frac{1}{Q} y_{3}, \\
t & =t^{\prime} \omega_{0}, \\
\omega_{0} & =\frac{1}{\sqrt{L \frac{C_{1} C_{2}}{C_{1}+C_{2}}}}
\end{aligned}
$$

where $x_{1}, x_{2}$, and $x_{3}$ belong to the master circuit, $y_{1}, y_{2}$ and $y_{3}$ belong to the slave circuit, $\omega_{0}$ is the resonant frequency of the tank circuit due to $L, C_{1}$ and $C_{2}$. The time derivative of $y_{2}$ containing the coupling term depends on variables of both circuits. The non linear terms are given by $n(x)=e^{-x}-1$, and $n(y)=e^{-y}-1$. The dimensionless parameters are

$$
\begin{aligned}
& Q=\frac{\omega_{0} L}{R}, \\
& k=\frac{C_{2}}{C_{1}+C_{2}}, \\
& g=\frac{I_{0} L}{V_{T} R\left(C_{1}+C_{2}\right)},
\end{aligned}
$$

and $\varepsilon$ is the coupling strength, that is defined as $\varepsilon=\sqrt{L / C_{1}} R^{*-1}$. We use $k=0.5$, $\alpha_{F}=0.996, R=80 \Omega, C_{1}=C_{2}=1 \mu F, L=18.2 \mu H, V_{T}=27 m V$, and $Q=1.77$. With these value set and $g=2.863$ the Colpitts oscillator presents chaotic behaviour. The system presents a broad range of different dynamical regimes as the parameter $g$ is varied, such as chaotic behaviour, periodic solutions, hopf bifurcation, and coexistence 

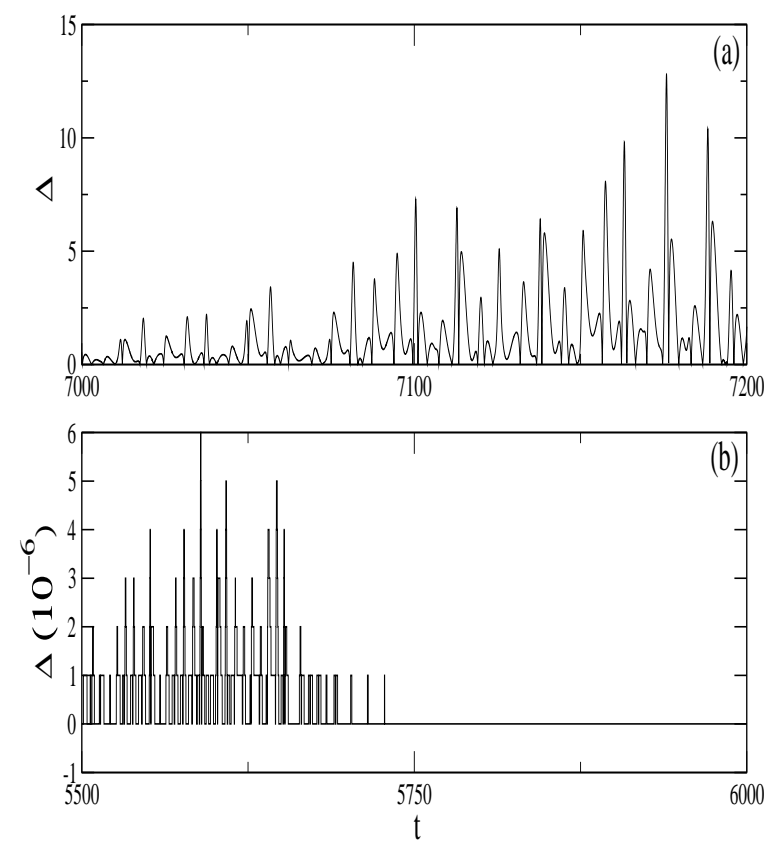

Figure 3. Time evolution of the synchronisation error considering (a) $\varepsilon=0.05$ and (b) $\varepsilon=0.089$.

of solutions. In this work, in order to study chaos synchronisation, we consider a small interval of the values of $g$ such that the system only presents chaotic behaviour.

\section{Chaos synchronisation}

Chaotic systems have applications in secure and spread spectrum communications. Previous works presented applications of chaos synchronisation in wireless communications [18] and multiplexing mixed chaotic signals generated by different electronic oscillators [19]. Chaos synchronisation occurs when the state variables of both circuits are equal. Such condition is achieved after a transient time that depends on the stability of the synchronisation manifold.

A numerical diagnosis to state about the synchronous state is provided by the synchronisation error

$$
\Delta=\left|x_{1}-y_{1}\right| .
$$

Figure 3 shows the time evolution of $\Delta$ for two situations: (a) when there is no synchronisation between the Colpitts oscillators and (b) when there is chaos synchronisation. When the oscillators are completely synchronised we have $\Delta=0$.

We investigate the dependence of the synchronisation error on the control parameter $g$ and the coupling strength $\varepsilon$. Using the time averaged error

$$
\bar{\Delta}=\frac{1}{t_{2}-t_{1}} \sum_{t_{1}}^{t_{2}} \Delta(t),
$$




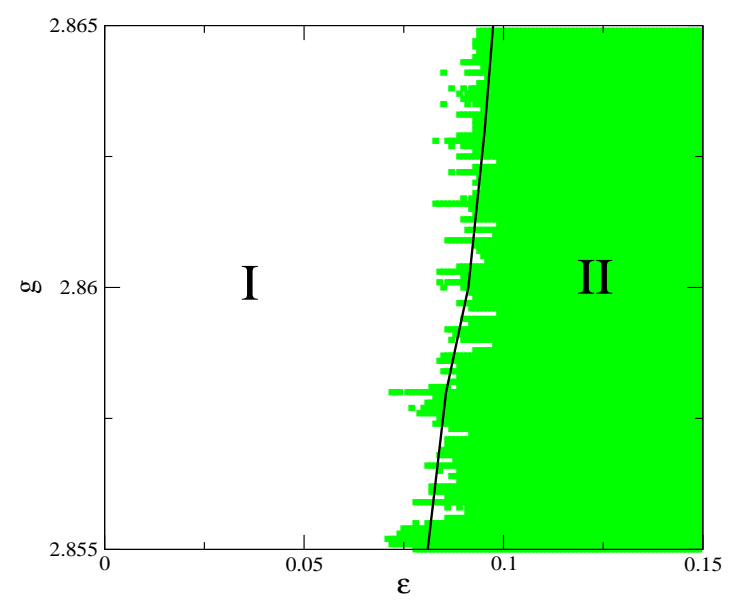

Figure 4. (Colour online) Synchronised domains (green region) in parameter plane $g \times \varepsilon$. The black line separates the regions for which the synchronisation manifold is unstable (I) and stable (II). Stability is measured by the Lyapunov exponents of the synchronisation manifold.

where $t_{2}-t_{1}$ is the time window for measurements, chaos synchronisation is stated when $\bar{\Delta}<10^{-4}$. We consider $t_{1}=5000$ and $t_{2}=10000$ but similar results were obtained for $t_{1}=18000$ and $t_{2}=20000$. Figure 4 shows a parameter space indicating by colours regions of parameters that lead to no synchronisation (white) and regions of parameters that lead to synchronisation (green), representing parameters for which $\bar{\Delta}<10^{-4}$.

We calculate the spectrum of Lyapunov exponents of the synchronisation manifold and its transversal directions in order to verify the local stability of the synchronisation manifold. We obtain the spectrum considering the same initial conditions for both circuits: $x_{1}=y_{1}=0.02, x_{2}=y_{2}=10^{-4}$ and $x_{3}=y_{3}=10^{-4}$. We are interested in the two largest Lyapunov exponents. When the maximal exponent is positive and the second largest is negative the system presents chaos synchronisation [20]. The synchronisation manifold is locally stable, since the negative exponent measures how perturbation propagate along the direction transversal to the synchronisation manifold. Consequently, the circuits can synchronise. The black line, shown in Fig. 4, separates two regions. In region I the coupled oscillators do not synchronise, while in region II synchronisation occurs.

We can see in Fig. 4 that the boundary between the two regions has an irregular pattern. This suggests the existence of an entangled basin boundary for the synchronous attractor [21]. Consequently, the time to reach the synchronous state will strongly depend on the initial conditions, some of them responsible for very long transients. The transient time is denoted by $\tau$, and its average value is $\tau_{M}$.

The histogram of the transient time for an ensemble of initial conditions is shown in Fig. 5, where the red circles correspond to $\varepsilon=0.090$ and the black squares to $\varepsilon=0.086$. The statistical distribution of the transient sizes was obtained through $10^{4}$ different initial conditions of $x_{2}$ and $y_{2}$, showing that small transients are more common 


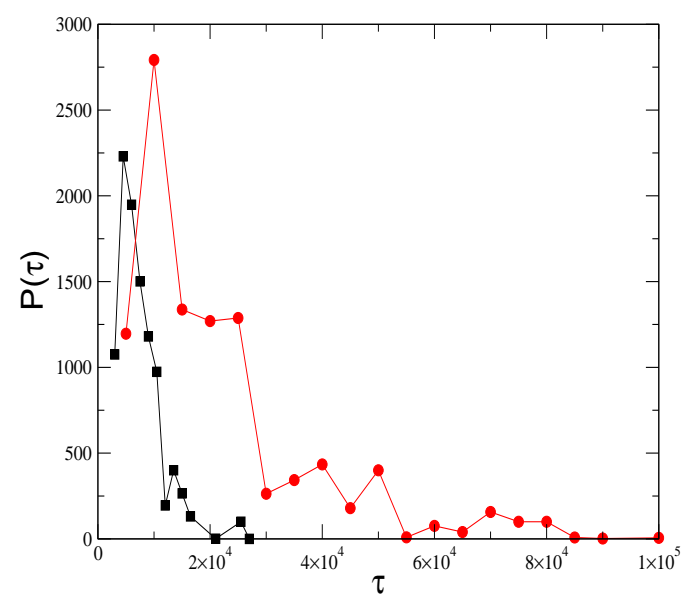

Figure 5. (Colour online) Histogram of the transient time intervals for a total of $10^{4}$ different initial conditions $x_{2}$ and $y_{2}$ in the interval $[0,0.001]$, where we consider $g=2.863, \varepsilon=0.086$ (red circles) and $\varepsilon=0.090$ (black squares).

when the value of the coupling strength increases. Therefore, for some values of $g$ and $\varepsilon$, around the region of the boundary in the green area of Fig. 4, the circuits may present large transient depending on the initial conditions.

Here we recall that there is a distinct class of chaotic transients that is referred to as super persistent [13, 14]. This transient times is characterized by the following scaling law for their average transient

$$
\tau_{M} \sim \exp \left[\beta\left(p-p_{c}\right)^{-\gamma}\right]
$$

where $\beta$ and $\gamma$ are positive constants, $p_{c}$ is a critical parameter value, and the transient occurs for $p>p_{c}$. The least-squares fit in Fig. 6] exhibits an exponential distribution for $\tau_{M}$ and $\varepsilon-\varepsilon_{c}$, which indicates a super persistent transient with exponent $\gamma=1$.

\section{The effect of noise}

We analyse chaos synchronisation under realistic conditions, due to noise in experiments [22]. We add a stochastic perturbation in the variable $y_{2}(t)$ of equation (6)

$$
\frac{d y_{2}}{d t}=\frac{g}{Q k}\left[\left(1-\alpha_{F}\right) n\left(y_{2}\right)+y_{3}\right]+\varepsilon\left[x_{2}-y_{2}\right]+A r(t)
$$

where $A$ is the level of the stochastic perturbation and $r(t)$ is a pseudo-random variable. We consider a random number generator that returns a normally distributed deviate with zero mean and unit variance. In other words, the noise is a white Gaussian noise $(\mathrm{AWGN})[23]$.

To understand the effect of noise in the synchronisation conditions, we measure the synchronisation error for different values of noise level and coupling strength. In Fig. 7 , we plot the time averaged synchronisation error versus the coupling strength for three values of the noise level. When the noise level is small (green circles) the value of the 


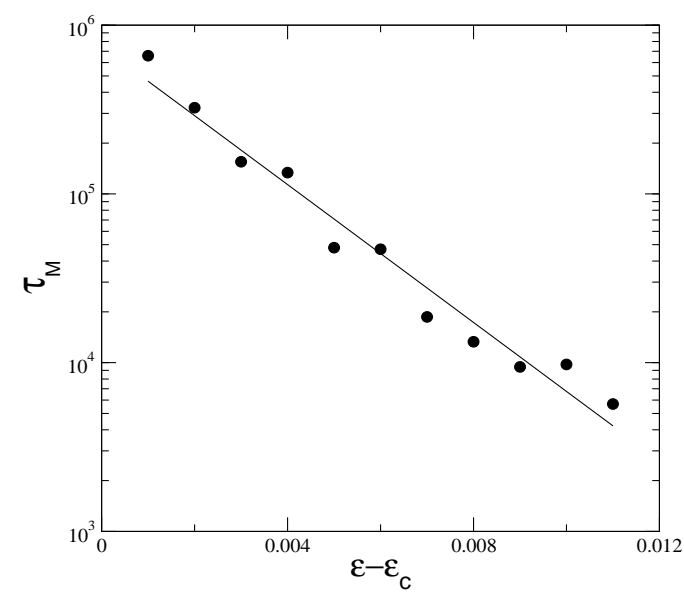

Figure 6. Scaling of super persistent transient varying the coupling strength for $g=2.863$ and $\varepsilon_{c}=0.078$. Each point represents the average over 100 different initial conditions of $x_{2}$ and $y_{2}$. The solid line is an exponential fitting with exponent -470.31 . The value of the $\varepsilon_{c}$ is obtained when the synchronisation manifold becomes stable.

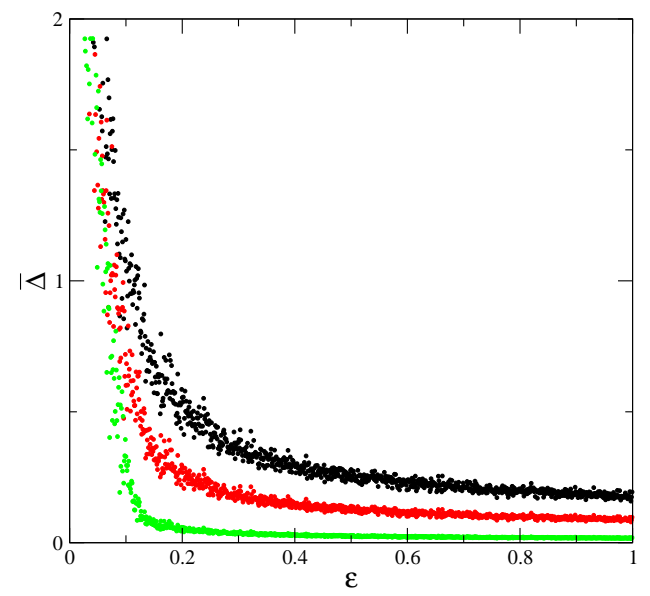

Figure 7. (Colour online) Synchronisation error versus coupling strength for $g=$ 2.863, $A=0.1$ (green circles), $A=0.5$ (red circles) and $A=1.0$ (black circles). These three cases have exactly the same initial conditions.

time averaged error decreases sharply when the coupling strength increases. For larger values of $A$ (red and black circles) the time averaged error decays less abruptly as a function of $\varepsilon$. For all situations, $\bar{\Delta}>0$, showing that synchronisation is suppressed.

Synchronisation is affected by noise. In Fig. 8 we consider the same parameters used in Fig. 4 but add noise with level $A=3 \times 10^{-5}$. Comparing Fig. 8 (with noise) with Fig. 4 (without noise) it is possible to observe that due to the effect of the noise, a larger value of $\varepsilon$ is necessary to make the systems synchronise. Similar to Fig. 4, the structure of the boundary between the synchronous and the non-synchronous region is a consequence of the existence of entangled basin boundary that produces super persistent transients. We obtain the synchronised region by verifying if $\bar{\Delta}<10^{-4}$, for $t_{1}=1000$ 


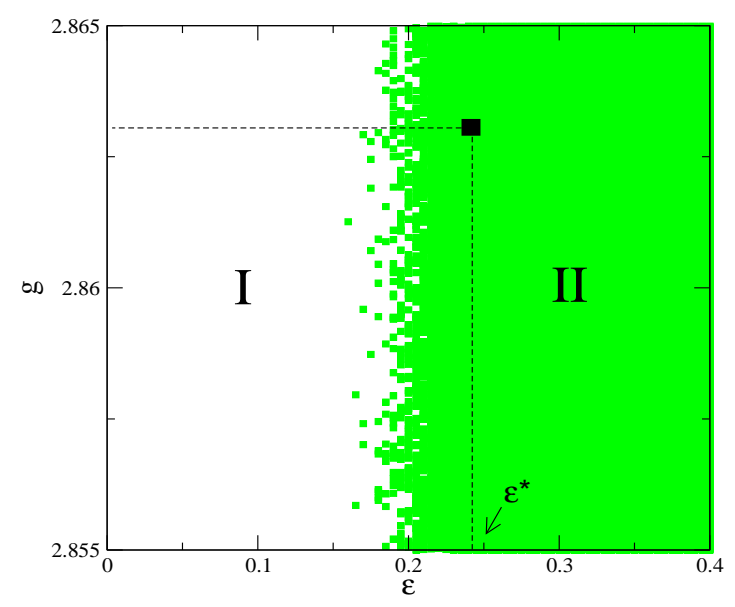

Figure 8. (Colour online) Synchronised domains (green region) considering the same parameters according to Fig. 4, and a small noise $A=3 \times 10^{-5}$.

and $t_{2}=20000$. We analyse the noise effect on the synchronised region fixing the value of $g$ and varying the noise amplitude $A$ to obtain the $\varepsilon^{*}$ in that the average transient $\tau_{M}$ is approximately $10^{4}$ (Fig. 9a). Therefore, the larger the noise amplitude is, the larger the coupling strength must be to synchronise.

The phenomenon of super persistent transient is effected by the noise, that is, a small noise increases the transient times. Fig. 9(b) shows histograms for $g=2.863$, $\varepsilon=1, A=5 \times 10^{-5}$ (black circles), and $A=6 \times 10^{-5}$ (red squares), in order to show that the noise induces longer transients. When $A \leq 4 \times 10^{-5}$ the transients have values around $10^{3}$. Moreover, for $A \geq 7 \times 10^{-5}$ the noise may suppress chaos synchronisation. It is worth to comment that near the critical parameter $\varepsilon_{c}$, the average transient $\tau_{M}$ quickly increases when the noise amplitude grows, according to an exponential relationship $\tau_{M} \sim \phi \exp (\varphi A)$, where $\phi$ and $\varphi$ are positive constants.

\section{Conclusions}

In this paper, we studied some aspects of chaos synchronisation displayed by two coupled Colpitts with master-slave configuration. We obtained a set of parameters which may lead the coupled circuits to a synchronised or non synchronised state. We verified the existence of super persistent transients. This transient is mainly situated in the border of the synchronised domain in the parameter space $g$ versus $\varepsilon$, where $g$ is the loop gain of the oscillator, and $\varepsilon$ is the strength coupling.

The effect of noise on the coupled circuits were considered. Noise acts on the system in a way that synchronisation can only be achieved for higher coupling strength. Moreover, the transients become longer.

Our results enable us to predict a set of parameters of the coupled Colpitts oscillators to observe super persistent transients that can be used in laboratory experiments. The described persistent transients are similar to those observed in 


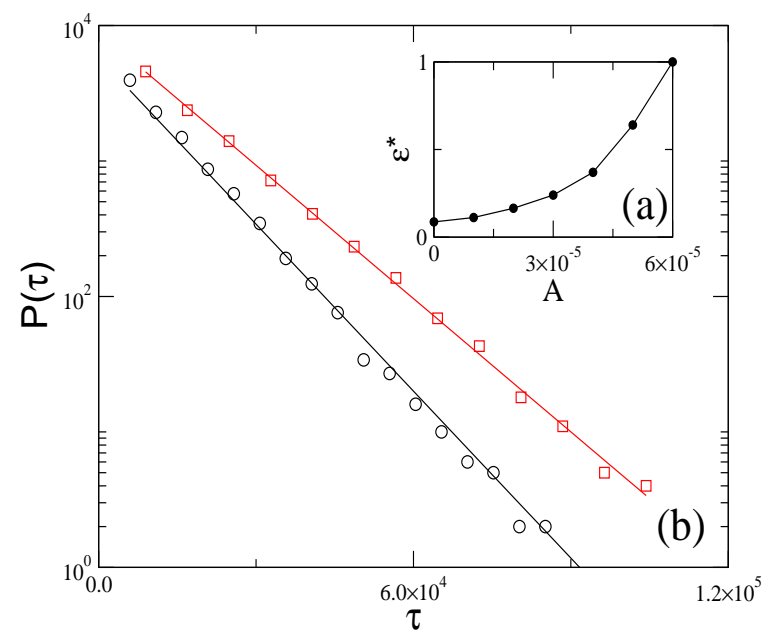

Figure 9. (Colour online) (a) $\varepsilon^{*}$ versus $A$, where the black circles correspond to $\tau_{M} \approx 10^{4}$. (b) Histograms for transient time considering $g=2.863, \varepsilon=1, A=5 \times 10^{-5}$ (black circles), and $A=6 \times 10^{-5}$ (red squares).

dissipative systems, and should be related to the chaotic saddle of the coupled systems [24, 25].

In future works, we plan to study this coupled system considering electronic simulations [26], and also to get experimental results through an electronic circuit.

\section{Acknowledgments}

This work was possible by financial support from following Brazilian government agencies: CNPq, CAPES, Fundação Araucária (Paraná) and FAPESP. M. S. Baptista acknowledges EPSRC-EP/I032606/1.

\section{References}

[1] Pecora L M and Carrol T L 1990 Synchronization in chaotic systems Phys. Rev. Lett. 64(8) 821824

[2] Batista C A S, Lameu E L, Batista A M, Lopes S R, Pereira T, Zamora-López G, Kurths J and Viana RL 2012 Phase synchronization of bursting neurons in clustered small-world networks Phys. Rev. E 8(6) 016211

[3] Yang J Q and Zhu F L 2013 Synchronization for chaotic systems and chaos-based secure communications via both reduced-order and step-by-step sliding mode observers Commun. Nonlinear Sci. Numer. Simulat. 18 926-937

[4] Wang J-W and Chen A-M 2010 Partial synchronization in coupled chemical chaotic oscillators $J$. Comp. Appl. Math. 233(8) 1897-1904

[5] Suykens J A K, Curran P F and Chu L O 1999 Robust synthesis for masterslave synchronization of Lure system IEEE Trans. Circuits Syst. I Fundam. Theory Appl. 46(7) 841

[6] De Feo O, Maggio G M and Kennedy M P 2000 The Colpitts oscillator: families of periodic solutions and their bifurcations Int. J. Bifurc. Chaos 10(5) 935-958

[7] De Feo O and Maggio G M 2003 Bifurcations in the Colpitts oscillator: from theory to practice. Int. J. Bifurc. Chaos 13(10) 2917-2934 
[8] Li H G, Zhou S P and Yang K 2007 Controlling chaos in Colpitts oscillator Chaos Solitons Fractals 33 582-587

[9] Effa J Y, Essimbi B Z and Ngundam J M 2009 Synchronization of improved chaotic Colpitts oscillators using nonlinear feedback control Nonlinear Dyn. 58 39-47

[10] Baziliauskas A, Krivickas R and Tamasevicius A 2006 Coupled chaotic Colpitts oscillators: Identical and mismatched cases Nonlinear Dyn. 44 151-158

[11] Mata-Machuca J L and Martínez-Guerra F 2012 Asymptotic synchronization of the Colpitts oscillator Comp. Math. Appl. 63 1072-1078

[12] Kengne J, Chdjou J C, Kenne G and Kyamakya K 2012 Dynamical properties and chaos synchronization of improved Colpitts oscillators Commun. Nonlinear Sci. Numer. Sim. 17 29142923

[13] Grebogi C, Ott E and Yorke J A 1985 Super persistent chaotic transients Ergod. Th. Dynam. Sys. 5(3) 341-372

[14] Andrade V and Lai Y-C 2001 Super persistent chaotic transients in physical systems: effect of noise on phase synchronization of coupled chaotic oscillators Int. J. Bifurc. Chaos 11(10) 2607-2619

[15] de Souza S L T, Caldas I L, Viana R L, Batista A M and Kapitaniak T 2005 Noise-induced basin hopping in a gearbox model Chaos Solitons Fractals 26(5) 1523-1531

[16] de Souza S L T, Batista A M, Caldas I L, Viana R L and Kapitaniak T 2007 Noise-induced basin hopping in a vibro-impact system Chaos Solitons Fractals 32(2) 758-767

[17] Kennedy M P 1994 Chaos in the Colpitts oscillator IEEE Trans. Circuit Syst. 41 771-774

[18] Sano E 2006 A chaotic wireless communication system based on collective synchronization among wireless nodes IEICE Electronics Express 3(12) 262268

[19] Zhi-Guo S, Li-Xin R and Kang-Sheng C 2005 Multiplexing chaotic signals generated by Colpitts oscillator and Chua circuit using dual synchronization Chin. Phys. Lett. 22(6) 1336-1339

[20] Gade P M, Cerdeira H A and Ramaswamy R 1995 Coupled maps on trees Phys. Rev. E 52 $2478-2485$

[21] Camargo S, Viana R L and Anteneodo C 2012 Intermingled basins in coupled Lorenz systems Phys. Rev. E 85036207

[22] Hong S, Shi Z, Wang L, Gu Y and Chen K 2012 Adaptive regularized particle filter for synchronization of chaotic Colpitts circuits in an SWGN channel Circuits Syst. Signal Process. DOI 10.1007/s00034-012-9506-y 1-17

[23] Shi Z, Hong S and Chen K 2008 Experimental study on tracking the state of analog Chua's circuit with particle filter for chaos synchronization Phys. Lett. A 372 5575-5580

[24] Kraut S and Feudel U 2002 Multistability, noise, and attractor hopping: The crucial role of chaotic saddles Phys. Rev. E 66 015207(R)

[25] Kraut S and Feudel U 2003 Noise-induced escape through a chaotic saddle: lowering of the activation energy Physica D 181 222-234

[26] Kenfack G and Tiedeu A 2013 Secured transmission of ECG signals: Numerical and electronic simulations J. Signal Inf. Process. 4 158-169 